\title{
Is Periodontitis Associated with Decreased Glomerular Filtration Rate, with Oxidative Stress as an Important Link?
}

\author{
${ }^{1}$ Meenakshi Sreeram, ${ }^{2}$ Suryakar AN, ${ }^{3}$ Dani NH, ${ }^{4}$ Kulkarni MB \\ 1 Dept of Biochemistry, MGV's KBH Dental College \& Hosp, Nasik/MUHS, Nasik/Maharashtra,India. \\ 2 Registrar, Maharashtra University of Health Sciences (MUHS), Nasik/ Maharashtra, India. \\ ${ }^{3}$ Professor \& Head, Dept. Of Periodontics, MGV's KBH Dental College\& Hosp, Nasik/Maharashtra, India \\ ${ }^{4}$ Head of Department of Mathematics \& Statistics, BYK (Sinnar) College, Nasik/Maharashtra, India
}

\begin{abstract}
Context: The prevalence of Chronic Kidney Disease is increasing world-wide. Besides traditional risk factors, many non-traditional risk factors may be responsible for development of the disease. Aims: To see if chronic generalized periodontitis is associated with decreased glomerular filtration rate; with oxidative stress as an important link. Design: A cross-sectional study involving 300 individuals, 150 cases and 150 controls. Setting: Candidates enrolled were patients visiting the OPD of MGV's Dental College \& Hospital, Nasik, India between Jan 2011 and Dec 2012. Materials and Methods: Serum/plasma and urine samples of patients with/without periodontitis were analyzed for marker of inflammation (CRP), kidney function tests (serum creatinine, eGFR, serum Urea and ACR) and markers of oxidative stress (MDA, GGT, Uric Acid and TAC). Statistical Analysis used: Analysis was done using Statistical software SPSS version 20. Values were expressed as mean \pm standard deviation. $P<0.05$ was considered significant. Results: Patients with periodontitis were found to have higher mean CRP, lower eGFR, higher serum urea, higher MDA, GGT and lower uric acid and TAC as compared to controls. Conclusions: Patients with periodontitis may be at an increased risk for development of CKD. Identification of such patients may help in early diagnosis of the disease.
\end{abstract}

Key Words: Periodontitis, Chronic Kidney Disease, C-reactive protein, Oxidative Stress, Glomerular Filtration Rate.

\section{Introduction}

Since ancient times, diseases of the oral cavity, especially periodontal diseases, have always been considered one the most important global health burdens and continue to plague people from both, the developed and developing countries alike. Chronic Kidney Disease (CKD) and its concomitant sequelae represent a major challenge to the medical world. These two diseases together pose the most challenging health issues for the human population.

In the past two decades, the emerging field of periodontal medicine has investigated the link between periodontitis and other systemic diseases. There has been enough evidence to suggest that periodontal infections can adversely affect systemic health with manifestations such as atherosclerotic vascular disease, diabetes, preeclampsia, metabolic syndrome, etc. ${ }^{[1-5]}$

In the last decade, accumulated evidence seems to be pointing towards an association between periodontitis and CKD. ${ }^{[6-9]}$ Many studies have proved without a reason of doubt that periodontitis is an inflammatory disease that is characterized by increased oxidative stress. ${ }^{[10-11]}$ It is also known that CKD too is an inflammatory syndrome associated with adverse outcomes. ${ }^{[12]}$ Oxidative stress has been implicated in the development of almost all of the major degenerative diseases we have today like cancer, heart disease, stroke, diabetes, arthritis, Parkinson's, Alzheimer's, asthma \& emphysema, liver and kidney diseases and many more. CKD too is a pro-oxidant state. ${ }^{[13]}$ Relative importance of each type of oxidant involved has not been sufficiently evaluated. Massy et al feel that the prevalence of oxidative stress among CKD patients remains undetermined due to lack of substantial population based studies in this regard. ${ }^{[14]}$

Scientists have been harvesting increasing evidence that periodontal pathogens are significantly associated with impaired kidney functions independent of traditional risk factors and that periodontitis predicts the development of over nephropathy and ESRD in individuals with type-2 diabetes. ${ }^{[15-16]}$ On the other hand, the consensus among some other scientists is that severe periodontitis is not associated with alterations in kidney functions. ${ }^{[17-18]}$ A systematic review published in 2013 says that $80 \%$ of the observational studies reported some degree of association while all interventional studies found positive effect of periodontal treatment on eGFR. ${ }^{[19]}$

In this maze of information, it is impalpable to figure out the whether or not there is an association between these two diseases. Even studies showing association between periodontitis and CKD conclude that a lot more needs to be done to understand the true nature of the link between the two. Oxidative stress could be the crucial connecting link between the two conditions. If such an association does exist and the missing pieces of this puzzle are put together, it would open up a very exciting possibility for early detection and treatment of 
people at high risk for developing CKD. The topic is also relevant for a country like India which has a considerable proportion of CKD cases and new cases are being added at an alarming rate. There have hardly been any significant studies in this population exploring this possibility.

We decided to test the hypothesis that periodontitis is associated with decreased kidney functions with oxidative stress as the underlying common link. To this end, this study was designed to assess the association between periodontitis and kidney function markers, markers of inflammation and oxidative stress in otherwise systemically healthy subjects.

\section{Materials And Methods}

This cross-sectional study was conducted between Jan 2011 to Jun 2012 after the protocol was approved by the Institutional Ethics Committee of MGV's KBH Dental College and Hospital, Nasik and Human Ethics Committee, UDIRT, MUHS, Nasik. Prior to enrolment in the study, prospective candidates were given detailed oral information about the study. A Patient Information Sheet (PIS) was handed over to them and written informed consent obtained.

150 subjects in the age group of 18-70 years with chronic periodontitis and 150 healthy controls satisfying the inclusion and exclusion criteria became part of the study. They were recruited from the Out Patient Department of MGV's KBH Dental College \& Hospital, Nashik.

\subsection{Periodontal parameters:}

A full-mouth periodontal examination was performed by a single examiner (N.D.). The following periodontal parameters were assessed using a graduated UNC15 probe at 6 sites per tooth i.e. mesio-buccal, mid-buccal, disto-buccal, mesio-lingual, mid-lingual and disto-lingual: a. Probing Pocket Depth (PPD) b. Clinical Attachment Loss (CAL) c. Bleeding on Probing (BOP)

\subsection{Inclusion criteria:}

\subsubsection{Control group (CG)}

a. Included both, periodontally healthy and patients with gingivitis.

b. At least 14 teeth in mouth.

c. BOP at sites with PPD of 1-3mm.

d. Not barred by exclusion criteria.

\subsubsection{Study Group (SG) (Patients with Periodontitis)}

a. At least 14 teeth in mouth.

b. CAL of $1 \mathrm{~mm}$ or more with BOP, at more

than $30 \%$ of all sites in the mouth.

c. Not barred by exclusion criteria.

\subsection{Exclusion criteria:}

a. Unfavourable systemic conditions (rheumatic fever, heart problems, diabetes, hypertension, liver or kidney diseases, etc).

b. Any infection requiring prophylactic antibiotic treatment.

c. Pregnant and lactating women, women on hormonal contraceptives or on HRT

d. Patients taking steroids or NSAID (for previous 3 months) or vitamin supplements.

e. Persons who have undergone scaling/root planing in the past 6 months.

f. Smokers and subjects consuming alcohol.

\subsection{Physical Parameters:}

Physical examinations included weight, height and BMI assessments.

\subsection{Biochemical Parameters:}

$5 \mathrm{ml}$ blood samples was collected by venipuncture from the median cubital vein of which, about $4 \mathrm{ml}$ was transferred in plain bulb and $1 \mathrm{ml}$ in heparinised anticoagulant bulb. Samples for plasma collection were immediately processed (centrifuged at 3000rpm for 15 minutes) whereas the samples for serum collection were allowed to clot at room temperature before centrifugation. These were analyzed the same day for the following parameters:

1. Serum Creatinine estimation. (Modified Jaffe's Method using Kit from Yucca diagnostics, India)

2. Serum Urea(Urease method using kits from Accurex Biomedical Pvt. Ltd., India)

3. C- reactive protein (CRP) estimation (Immunoturbidometric Method, Quantia CRP UV, Tulip Diagnostics (P) Ltd. 
4. Estimation of serum lipid peroxide level (Malondialdehyde) by the method of Kei Satoh. ${ }^{\text {[20] }}$

5. GGT estimation by using diagnostic kit from Aspen Laboratories, India.

6. Uric acid estimation (Uricase method by using diagnostic kit from Accurex Biomedical Pvt. Ltd., India)

7. Total Antioxidant Capacity measured in plasma by using FRAP method by Benzie et al. ${ }^{\text {[21] }}$

Urine samples were collected and used for estimating following parameters:

1. Urinary Albumin (Pyrogallol Red Method, End Point by In-vitro diagnostic kit manufactured by TransAsia Bio-Medicals Ltd., in technical collaboration with ERBA Diagnostics Mannheim, Germany)

2. Urinary Creatinine (Modified Jaffe's Method using kits from Yucca Diagnostics, India)

\subsection{Calculations:}

1. Weight in kilograms and height in metres was measured and it was used to calculate BMI expressed as $\mathrm{Wt}(\mathrm{Kg}) / \mathrm{ht}\left(\mathrm{m}^{2}\right)$.

2. Estimated Glomerular Filtration Rate (eGFR) calculation using MDRD study equation. ${ }^{[22]}$ Renal insufficiency was defined as eGFR less than $60 \mathrm{ml} / \mathrm{min} / 1.73 \mathrm{~m}^{2}$.

3. Albumin to Creatinine Ratio (ACR) expressed in $\mathrm{mg} / \mathrm{gm}$ using above values. Microalbuminuria was defined as ACR 30-300 mg/gm.

\subsection{Statistical analysis}

\section{Results}

Data was analyzed using computer software, Statistical Package for Social Sciences (SPSS) Version 20. Data was expressed as mean \pm standard deviation as well as percentage. To elucidate associations and comparisons between different parameters, student's $t$ test was used and probability values of $<0.05$ were considered to be statistically significant.

Table 1: Demographic Characteristics of Controls and Cases

\begin{tabular}{|l|l|l|l|}
\hline Parameter & Controls & Cases & P value \\
\hline No of cases & 150 & 150 & \\
\hline Females & 30 & 36 & \\
\hline Males & 120 & 114 & \\
\hline Mean Age (Years & $34.2 \pm 12.0$ & $41.0 \pm 12.2$ & $<0.001^{*}$ \\
\hline Mean BMI & $22.12 \pm 3.72$ & $24.09 \pm 4.22$ & $<0.001^{*}$ \\
\hline
\end{tabular}

Table 2: Distribution of subjects in various classes based on BMI

\begin{tabular}{|c|c|c|c|c|c|c|c|c|c|c|c|c|}
\hline \multirow[t]{2}{*}{ BMI } & \multicolumn{2}{|c|}{$\begin{array}{c}18.5 \text { or less } \\
\text { Under } \\
\text { weight } \\
\end{array}$} & \multicolumn{2}{|c|}{$\begin{array}{c}18.5-24.99 \\
\text { Normal weight }\end{array}$} & \multicolumn{2}{|c|}{$\begin{array}{c}25-29.99 \\
\text { Over } \\
\text { weight } \\
\end{array}$} & \multicolumn{2}{|c|}{$\begin{array}{c}30-34.99 \\
\text { Obesity } \\
\text { (Class I) } \\
\end{array}$} & \multicolumn{2}{|c|}{$\begin{array}{c}35-39.99 \\
\text { Obesity } \\
\text { (Class II) } \\
\end{array}$} & \multicolumn{2}{|c|}{$\begin{array}{c}\geq 40 \\
\text { Morbid } \\
\text { Obesity } \\
\end{array}$} \\
\hline & No. & $\%$ & No. & $\%$ & No. & $\%$ & No. & $\%$ & No. & $\%$ & No. & $\%$ \\
\hline Controls & 25 & 16.66 & 91 & 60.66 & 31 & 20.66 & 03 & 02 & nil & 0 & nil & 0 \\
\hline Cases & 16 & 10.66 & 69 & 46 & 52 & 34.66 & 12 & 08 & 01 & 1.33 & nil & 0 \\
\hline
\end{tabular}

Table 3: Comparison of Mean values of Various Parameters in Controls and Cases

\begin{tabular}{|l|l|l|l|}
\hline Parameter & Controls & Cases & P value \\
\hline Mean CRP(mg/dl) & $0.28 \pm 0.37$ & $1.62 \pm 1.17$ & $<0.001^{*}$ \\
\hline Mean eGFR(ml/min/1.73m $\left.{ }^{2}\right)$ & $111.55 \pm 21.4$ & $84.35 \pm 17.8$ & $<0.001^{*}$ \\
\hline Mean Urea(mg/dl) & $23.42 \pm 3.89$ & $31.04 \pm 6.27$ & $<0.001^{*}$ \\
\hline Mean MDA(nmol/ml) & $3.28 \pm 0.52$ & $5.31 \pm 0.86$ & $<0.001^{*}$ \\
\hline Mean GGT(U/L) & $18.08 \pm 4.30$ & $29.75 \pm 8.68$ & $<0.001^{*}$ \\
\hline Mean Uric Acid(mg/dl) & $4.92 \pm 0.87$ & $4.29 \pm 1.15$ & $<0.001^{*}$ \\
\hline Mean TAC(mmol/lit) & $2.38 \pm 0.13$ & $1.99 \pm 0.17$ & $<0.001^{*}$ \\
\hline ACR $\geq 30 \mathrm{mg} / \mathrm{gm}$ & Nil & 08 & \\
\hline
\end{tabular}

*very significant

Table 4: Distribution of subjects in various classes based on eGFR(ml/min/1.73 $\mathrm{m}^{2}$ )

\begin{tabular}{|c|c|c|c|c|c|c|c|c|c|c|c|c|}
\hline \multirow{2}{*}{ eGFR } & \multicolumn{2}{|c|}{$>100$} & \multicolumn{2}{|c|}{$90-100$} & \multicolumn{2}{|c|}{$60-89$} & \multicolumn{2}{|c|}{$30-59$} & \multicolumn{2}{|c|}{$15-29$} & \multicolumn{2}{|c|}{$<15$} \\
\hline & No. & $\%$ & No. & $\%$ & No. & $\%$ & No. & $\%$ & No. & $\%$ & No. & $\%$ \\
\hline Controls & 103 & 68.66 & 23 & 15.33 & 24 & 16 & nil & 0 & nil & 0 & nil & 0 \\
\hline Cases & 28 & 18.66 & 26 & 17.33 & 91 & 60.66 & 05 & 3.33 & nil & 0 & nil & 0 \\
\hline
\end{tabular}


TABLE 1 compares the demographic characteristics between the two groups. There are a considerably lesser number of women as compared to men in both the groups. There is a significant difference in the mean ages of controls and cases indicating that periodontitis is associated with increasing age. The mean BMI of cases is higher than that of controls (24.09 versus 22.12), even though both values fall in the range of normal BMI. The mean values do not reveal the true picture. When patients are distributed into different categories based on BMI (TABLE 2), it is found that higher percentage of controls $(60.66 \%)$ have normal BMI as compared to cases (46\%). Likewise, $20.66 \%$ of controls are overweight as compared to $34.66 \%$ of overweight cases. $2 \%$ of the controls have Class I Obesity and there are none with Class II obesity as compared to $8 \%$ and $1.33 \%$ of cases with Class I and Class II obesity respectively

TABLE 3 gives the comparison of mean values of various parameters in controls and cases. Serum Creatinine values were incorporated in the MDRD study equation and used for calculating eGFR. Though this formula has not been standardized for the Indian population, this is the most popular and widely used formula for calculating eGFR in clinics all over India. Diagnosis of CKD and its treatment is based on this calculation. [23] The calculation of eGFR in our study population (TABLE 4) revealed that a very high percentage of healthy controls $(68.66 \%)$ have eGFR higher than $100 \mathrm{ml} / \mathrm{min} / 1.73 \mathrm{~m}^{2}$. Only $18 \%$ of the study group subjects have eGFR in this range. Similarly $60.66 \%$ of study group subjects have eGFR in the range of 60-89 $\mathrm{ml} / \mathrm{min} / 1.73 \mathrm{~m}^{2}$ as compared to $16 \%$ of the controls who have eGFR in this range. $3.33 \%$ of the study group subjects have eGFR in the range of $30-59 \mathrm{ml} / \mathrm{min} / 1.73 \mathrm{~m}^{2}$ as compared to no subject from the control group. Besides, 08 of the study group subjects $(5.33 \%)$ had ACR> $30 \mathrm{mg} / \mathrm{gm}$ indicating microalbuminuria as compared to none of the control group subjects. Within reference range, mean serum urea levels are also higher in patients with chronic generalized periodontitis as compared to controls (31.04 \pm 6.27 versus 23.42 \pm 3.89$)$.

In our study, we found that controls have significantly lower mean CRP values as compared to study group (0.28 \pm 0.37 versus $1.62 \pm 1.17)$. Mean MDA and GGT values are found to be significantly higher in the patients with periodontitis while mean uric acid levels and TAC are lower as compared to controls.

\section{Discussion}

It appears that patients with chronic generalized periodontitis are more likely to be associated with higher BMI as compared to periodontally healthy controls. Our results are consistent with similar views expressed in other studies. ${ }^{[24-25]}$ Ramos et al found a significant relationship between increasing adiposity and incidence of CKD. The resultant obesity may be an independent risk factor for CKD incidence and its progression to ESRD. ${ }^{[26]}$ Our results seem to indicate that patients with moderate to severe periodontitis have significantly higher BMI than controls thus putting them at a higher risk of developing CKD.

Chronic inflammation in the body is measured by the elevated levels of inflammatory markers including CRP, highly sensitive C-reactive protein (hs-CRP), interleukin-6, haptoglobin and fibrinogen. Low grade chronic inflammation causes endothelial cell activation which is an early event in atherogenesis which in turn poses increased risk of atherosclerotic cardiovascular disease. ${ }^{[27]}$ Elevated CRP damages vascular endothelium leading to microalbuminuria which is now recognized as the laboratory indicator of early renal damage due to endothelial dysfunction leading to more advanced kidney damage and decreased GFR. [28] Periodontitis also contributes to the proinflammatory burden of CKD. Recent studies have shown that successful periodontal treatment results in significant reductions in hsCRP, IL-6 and Prohepcidin levels in patients with CKD. ${ }^{[29]}$ Benefits with regard to serum CRP levels and WBC/ neutrophils are also obtained in otherwise healthy patients affected by severe generalized periodontitis. ${ }^{[30]}$

Inflammation causes reactive oxygen species (ROS) overproduction, which in turn recruit other inflammatory cells leading to additional ROS production thus amplifying the damage causing increased oxidative stress. ${ }^{[31]}$ This leads to increased lipid peroxidation causing increase in the levels of Malondialdehyde (MDA). ${ }^{[32]}$ All studies are unanimous in agreeing that periodontitis causes increased oxidative stress resulting in increased levels of MDA. Gupta M, et al also found similar trends in MDA in patients with chronic periodontitis. ${ }^{[33]}$

Significantly increased levels of GGT were found in patients with periodontitis as compared to controls. Though GGT is routinely used as a marker for liver functions, results of many studies in the recent times have touted GGT as a very good indicator of oxidative stress. ${ }^{[34-35]}$ Results of a prospective study by Ryu et al seem to indicate that serum GGT may be an early predictor for the development of CKD. ${ }^{[36]}$ Uric acid is a very important antioxidant in the serum and acts as a potent free- radical scavenger, inhibiting lipid peroxidation. ${ }^{[37]}$ In particular, it has been found to react with superoxide anion and peroxynitrite and also helps in maintaining levels of extracellular SOD by preventing its oxidative degradation. ${ }^{[38]}$ Lower levels of uric acid in patients with periodontitis would decrease their antioxidant defence, making them susceptible to the damaging effects of oxidative stress. This could also explain the higher levels of lipid peroxidation found in patients with periodontitis as compared to controls. The results of the present study are consistent with that of Mathur et al who also found decrease in the concentration of uric acid levels in saliva with increase in severity 
of periodontitis. ${ }^{[39]}$ Uric acid is positively associated with total plasma antioxidant capacity which represents the sum total of all the antioxidant defence mechanisms in the body. The balance between antioxidant defence and repair systems and pro-oxidant mechanisms of cell damage may be tipped in favour of tissue destruction either by increases in radical production or by a lowered anti-oxidant defence, the former of which has already been demonstrated in periodontitis. ${ }^{[40]}$ A significant decrease in the TAC values of cases indicates that the inflammatory burden of chronic periodontitis disturbs the delicate prooxidant- antioxidant balance in the body creating oxidative stress. Brock et al suggest that reduced plasma TAO defence could result from low-grade systemic inflammation induced by the host response to periodontal bacteria or may be an innate feature of patients with periodontitis. ${ }^{[41]}$

Patients with known history of diabetes and hypertension (major risk factors for CKD) have been excluded from the study. Using healthy/ gingivitis patients as reference group, these results therefore seem to indicate that patients with chronic generalized periodontitis have reduced kidney functions as compared to healthy controls. Kshirsagar et al too concluded in their studies that periodontitis is associated with renal insufficiency. ${ }^{[42]}$

In the developed countries, a major chunk of health care funds are used for management of End Stage Kidney Disease (ESRD). Over the past two decades, dialysis programs are growing at a rate of 6-12\% and don't give any indication of slowing down. The magnitude of the problem can be gauged by the fact that around the world, more than two million people now require renal replacement therapy (RRT) to sustain life, but this probably accounts for less than $10 \%$ of the people who need it. ${ }^{[43]}$

Data suggests that in USA alone, CKD continues to increase with more than 20 million affected individuals, many of whom are undiagnosed. ${ }^{[44]}$ In this country, 116,946 new ESRD patients were added in 2010 and the adjusted rate of incident ESRD is 348 per million population. ${ }^{[45]}$ This figure would be much higher in the poor and developing countries of the world where the under-privileged do not have access to even the basic medical amenities. The burden of CKD in terms of medical costs and shortened healthy life is onerous and the affected individuals have markedly increased risk of End-Stage Renal Disease (ESRD), Cardiovascular Disease (CVD) and premature death.

In a vast and developing country India, oral health and CKD are challenges which the country is simply not equipped to handle. The WHO Global Burden of the Disease Project says that the diseases of the kidney and urinary tract contribute to global burden with approximately 8,50,000 deaths every year and $115,010,107$ disability adjusted life years. It isn't hard to imagine the impact of CKD on human life when we say that it is the $12^{\text {th }}$ leading cause of death and the $17^{\text {th }}$ cause of disability globally ${ }^{\text {[46] }}$ It definitely appears that the resources and skill for taking care of this large case load, both in terms of personal and health care infrastructure do not exist currently in India and other developing countries.

Fisher et al ${ }^{[47]}$ say that there is a bidirectional relationship between CKD and periodontal disease mediated by hypertension and the duration of diabetes. But our studies show that after eliminating these major risk factors, patients with chronic generalized periodontitis may still be at an increased risk for developing CKD. The pathogenesis of periodontitis and CKD seems to overlap as a result of inflammation and increased oxidative stress. It is therefore very important to explore non-traditional risk factors like periodontitis and increased oxidative stress to identify people at higher risk for developing CKD. Conventional treatment of periodontitis may help in reducing the onset and progression of CKD and possibly assist in reducing the burden of this disease on the society. Effective periodontal treatment may also reduce the CRP levels in patients with a history of CKD and thus provide succour by decreasing the inflammatory burden in such patients. Efforts to decrease oxidative stress, addition of antioxidants to the treatment regime and understanding the molecular mechanisms of oxidative stress may be some of the approaches for future studies in this regard.

\section{Conclusions}

Our findings suggest that periodontal infections, possible through increased oxidative stress and an inflammatory pathway may be related to renal insufficiency and development of CKD. No studies in available literature have considered the association of periodontitis and chronic kidney disease using oxidative stress as a common link. This is definitely the first Indian study to report this kind of association. Stratification of chronic periodontitis may also be done to see how the severity of periodontal infection affects the various parameters. Other markers of oxidative stress may be evaluated to see how each one varies with the periodontal infection. Markers may be studied to evaluate the effect of periodontal treatment. Our results are consistent with previous studies and demonstrate strong biological plausibility. However, this study being cross-sectional in nature, we cannot demonstrate cause- effect relationship between periodontitis and CKD. But considering the high prevalence of periodontitis in the general population and the ever increasing burden of CKD, more longitudinal studies to gather concrete evidence are definitely warranted. 


\section{Acknowledgements}

The authors would like to express heartfelt thanks to all the participants in this study.

\section{References}

[1]. Papapanou PN, Trevisan M. Periodontitis and atherosclerotic vascular disease, What we know and why it is important. The Journal of the American Dental Association, 143(8), 2012, 826-28.

[2]. Ide M, Papapanou PN. Epidemiology of association between maternal periodontal disease and adverse pregnancy outcomesSystematic review, J Clin Periodontol, 40(suppl s 14), 2013,S181-S194.

[3]. Preshaw PM, Alba AL, Herrera D, Jepsen S, Konstantinidis A, Makrilakis K, Taylor R. Periodontitis and diabetes: a two- way relationship, Diabetologia, 55(1), 2012: 21-31

[4]. Anil S, Al Ghamdi HS. The impact of periodontal infections on systemic diseases. An update for medical practitioners, Saudi Med $J, 27(6), 2006,767-76$.

[5]. Bullon P, Morillo JM, Ramirez-Tortosa MC, Quiles JL, Newman HN, Battino M. Metabolic syndrome and periodontitis: Is oxidative stress a common link. J. Dent Res., 88(6), 2009, 503-18.

[6]. Pradeep AR, Kathariya R, Arjun Raju P, Sushma Rani R, Sharma A, Raghavendra NM. Risk factors for CKD may include periodontal diseases, as established by the correlations of plasma pentraxin-3 levels: a case control study, Int Uro Nephro, 44(3), 2012, 829-39.

[7]. Kshirsagar AV, Craig RG, Moss KL, Beck JD, Offenbacher S, Kotanko P, et al. Periodontal disease adversely affects the survival of patients with ESRD. Periodontal disease status affects ESRD mortality, Kidney International, 75, 2009, 746-51.

[8]. Ioannidou I, Swede H. Disparities in Periodontitis Prevalence among Chronic Kidney Disease Patients, J Dent. Res., 90(6), 2011, 730-34.

[9]. Fisher MA, Taylor GW, Shelton BJ, Jamerson KA, Rahman A, Ojo AO, Sehgal AR. Periodontal disease and other non-traditional risk factors for CKD, Am J Kidney Dis., 51(1), 2007, 45-52.

[10]. Borges I Jr, Moriera EA, Filho DW, de Oliveira TB, da Silva MB, Frode TS. Proinflammatory and Oxidative Stress Markers in patients with Periodontal Disease, Mediators Infalmm. 2007, 2007: 45794

[11]. D'Aiuto F, Nibali L, Parkar M, Patel K, Suvan J, Donos N. Oxidative Stress, systemic inflammation and Severe Periodontitis. $J$ Dent Res, 89(11), 2010, 1241-46.

[12]. Lacson E Jr, Lewin NW. C- Reactive Protein and End- Stage Renal Disease. Semin Dial., 17(6), 2004, 438-48.

[13]. Massy ZA, Stenvinkal P, Drueke TB. The role of Oxidative Stress in Chronic Kidney Disease. Semin Dial., 22(4), 2009, 405-08.

[14]. Zalba G, Fortuno A, Diez J. Oxidative Stress and Atherosclerosis in early Chronic Kidney Disease. Nephrol. Dial. Transplant., 21(10), 2006, 2686-90.

[15]. Kshirsagar AV, Offenbacher S, Moss KL, Barros SP, Beck JD. Antibodies to Periodontal organisms are associated with decreased kidney function. Blood Purif, 25, 2007, 125-32.

[16]. Shultis WA, Weil EJ, Looker HC, Curtis M, Shlossman M, Genco RJ, et al. Diabetes Care, 30 (2), 2007, 306-11.

[17]. Brotto RS, Vendramini RC, Brunetti IL, Marcantonio R AC, Ramos A PP, Pepato MT. Lack of correlation between Periodontitis and Renal Dysfunction in Systemically Healthy Patients. Eur J Dent, 5(1), 2011, 8-18.

[18]. Ziebolz D, Fischer P, Homecker E, Mausberg RF. Oral health of hemodialysis patients: a cross-sectional study of two German dialysis centers. Hemodial Int., 16(1), 2012, 69-75.

[19]. Chamborne L, Foz AM, Gugliemetti MR, Pannuti CM, Artese HP, Feres M, Ronito GA. Periodontitis and Chronic Kidney Disease: a systematic review of the association of diseases and the effect of periodontal treatment on eGFR. J Clin Periodontol., 40(5), 2013, 443-56.

[20]. Kei, Satoh, Serum lipid peroxide in cerebrovascular disorders determined by a new colorimetric method, Clinica Chimica Acta, 90, $1978,37-43$

[21]. Benzie IF, Strain JJ. The Ferric Reducing ability of plasma (FRAP) as a measure of "antioxidant power": the FRAP assay. Anal Biochem., 239(1), 1996,70-6.

[22]. Levey AS, Bosch JP, Lewis JB, Greene T, Rogers N, Roth D. A more accurate method to estimate glomerular filtration rate from serum creatinine: a new prediction equation. Modification of Diet in Renal Disease Study Group. Annals of Internal Medicine, 130(6), 1999, 461-7023

[23]. National Kidney Foundation. K/DOQI clinical practice guidelines for chronic kidney disease: evaluation, classification, and $\begin{array}{llllllll}\text { stratification. } & A m & J & \text { Kidney } & \text { Dis., } & 39 & \text { (Suppl } & 2 \text { ), }\end{array}$ http://www.kidney.org/professionals/kdoqi/guidelines ckd/p7 risk g15.htm Part7: Stratification of risk for progression of kidney disease and development of cardiovascular disease. Viewed 10 Feb 2013.

[24]. Wood N, Johnson RB, Streckfus CF. Comparison of body composition and periodontal disease using nutritional assessment techniques: Third National Health and Nutrition Examination Survey (NHANESIII). Journal of Clinical Periodontol., 30(4), 2003, 321-27.

[25]. Chaffee BW, Weston SJ. Association between chronic periodontal disease and obesity: A systematic review and meta-analysis. Journal of Periodontol., 81(12), 2010, 1708-24.

[26]. Ramos LF, Shintani a, Ikizler TA, Himmelfarb J. Oxidative stress and Inflammation are associated with adiposity in moderate to severe CKD. J Am Soc Nephrol., 19(3), 2008, 593-99.

[27]. Cleland SJ, Sattar N, Petrie JR, Forouhi NG, Elliott HL, Connell JM. Endothelial dysfunction as a possible link between C-reactive protein levels and cardiovascular disease. Clin Sci (Lond)., 98(5), 2000, 531-5

[28]. Kshirsagar AV, Bomback AS, Bang H, Gerber LM, Vupputuri S, Shoham DA, et al. Association of C-reactive protein and microalbuminuria (from the National health and Nutrition Examination Survey, 1999 to 2004). Am J Cardiol, 101, 2008, 401-06

[29]. Vilela EM, Bastos JA, Fernandes N, Ferriera AP, Choubah A, Bastos MG. Treatment of Chronic Periodontitis decreases serum Prohepcidin levels in patients with Chronic Kidney disease. Clinics, 66(4), 2011, 657-62.

[30]. Radafshar G, Shad B, Ariamajd E, Geranmayeh S. Effect of Intensive Non-Surgical Treatment on the Level of Serum Inflammatory Markers in Advanced Periodontitis. Journal of Dentistry, Tehran Univ of Medical Sciences, 7(1), 2010, 24-30.

[31]. Khansari N, Shakiba Y, Mahmoudi M. Chronic inflammation and Oxidative Stress as a Major Cause of Age-Related Diseases and Cancer. Recent Patents on Inflammation \& Allergy Drug Discovery, 3, 2009, 73-80.

[32]. Sreeram M, Suryakar AN, Kulhalli PM. A Study of Bio-Markers of Oxidative Stress and Inflammation in Chronic Kidney Disease. IOSR-JDMS, 11(2), 2013, 6-10.

[33]. Gupta M, Chari S, Kolte A, Chandankhede M. MDA levels in patients with chronic periodontitis. Journal of Evolution of Medical and Dental sciences, 2(24), 2013, 4325-28. 
[34]. Lee DH, Blomhoff R, Jacobs DR Jr. Is Serum Gamma Glutamyl Transferase a Marker of Oxidative Stress? Free Radic Res., 38(6), 2004, 535-9.

[35]. Pandur S, Pankiv s, Johannessen M, Moens U, Huseby NE. Gamma-Glutamyltransferase is upregulated after oxidative stress through the Ras signal transduction pathway in rat colon carcinoma cells. Free Radic Res., 41(12), $2007,1376-84$.

[36]. Ryu S, Chang Y, Kim DI, Kim WS, Suh BS. Gamma-Glutamyltransferase as a Predictor of CKD in Nonhypertensive and Nondiabetic Korean Men. Clin Chem., 53(1), 2007, 71-7.

[37]. Halliwell B, Gutteridge JMC, Free radicals in biology and medicine (Oxford: Clarendon Press, 1985).

[38]. Ejaz A Ahsan, Wei Mu, Duk-Hee Kang, Roncal C, Sautin Yuri Y, Henderson G, et al. Could uric acid have a role in acute renal failure? Clin J American Soc of Nephrol., 2(1), 2007, 16-21.

[39]. Mathur A, Mathur L, Manohar B, Shankarapillai R, Mathur H. Effect of antioxidants on progression of periodontal disease. International Journal of Dental Clinics, 3(4), 2011:13-15.

[40]. Chapple Iain LC. Role of free radicals and antioxidants in the pathogenesis of the inflammatory periodontal diseases. $J$ Clin Pathol: Mol Pathol., 49, 1996,M 247-M 255.

[41]. Brock GR, Butterworth CJ, Matthews JB, Chapple ILC. Local and Systemic Total Antioxidant Capacity in Periodontitis and Health. J Clin Periodontol., 31(7), 2004, 515-21.

[42]. Kshirsagar AV, Moss KL, Elter JR, Beck JD, Offenbacher S, Falk RJ. Periodontal disease is associated with renal insufficiency in the Atherosclerosis Risk in Communities (ARIC) study. Am J Kidney Dis., 45(4), 2005, 650-57.

[43]. Couser WG, RemuzziG, Mendis S, Tonelli M. The contribution of Chronic Kidney Disease to the Global Burden of Major Noncommunicable Diseases. Kid Int., 80(12), 2011, 1258-70.

[44]. National Chronic Kidney Disease Fact Sheet 2010. Available at www.cdc.gov/diabetes/pubs/factsheets/kidney.htm Accessed on 28 Jul 2013.

[45]. Atlas of Chronic Kidney Disease in the United States. Available at www.usrds.org/2012/pdf/v2 00intro 12.pdf. Accessed on 28 Jul 2013.

[46]. Prabhakar MR, Chandrasekaran V, Soundararajan P. Epidemic of chronic kidney in India- what can be done? Saudi J Kidney Dis Transpl., 19(5), 2008, 847-53.

[47]. Fisher MA, Taylor GW, West BT, McCarthy ET. Bidirectional relationship between Chronic Kidney disease and periodontal Disease: a study using structural equation modelling. Kidney Int., 79, 2011, 347- 55. 